Supporting Information for

\title{
IR and Vibrational Circular Dichroism Spectroscopy of Matrine- and Artemisinin-type Herbal Products: Stereochemical Characterization and Solvent Effects
}

\author{
Yuefei Zhang, ${ }^{\dagger}$ M. Reza Poopari, ${ }^{\ddagger}$ Xiaoli Cai, ${ }^{\ddagger}$ Aliaksandr Savin, ${ }^{\ddagger}$ Zahra Dezhahang, ${ }^{\ddagger}$ \\ Joseph Cheramy, ${ }^{\ddagger}$ and Yunjie $\mathrm{Xu}^{\ddagger} * *$ \\ ${ }^{\ddagger}$ Department of Chemistry, University of Alberta, Edmonton, Alberta, Canada, T6G 2G2 \\ ${ }^{\dagger}$ Permanent address: School of Chemistry and Environmental Engineering, Wuhan Institute of \\ Technology, Wuhan, Hubei, People’s Republic of China, 430073. \\ *Email: yunjie.xu@ualberta.ca
}

\section{Content:}

Table S1: Relative energies of the relevant stable conformers of matrine, oxymatrine, and

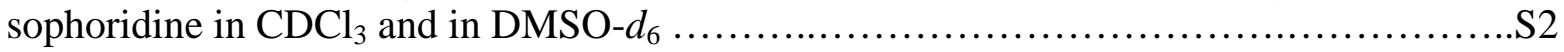

Figure S1: PES of dihydroartemisinin along the HOCO dihedral angle....

S2

Figure S2: Single conformer IR and VCD spectra of matrine in the gas phase, in $\mathrm{CDCl}_{3}$

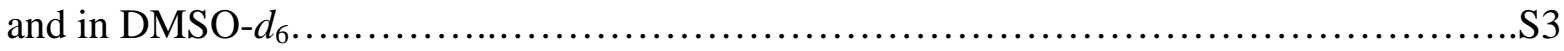

Figure S3: Single conformer IR and VCD spectra of oxymatrine in the gas phase, in

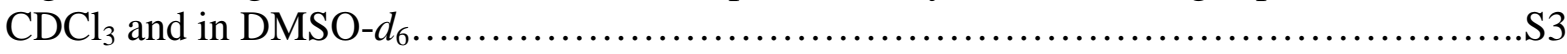

Figure S4: Single conformer IR and VCD spectra of sophoridine in the gas phase, in

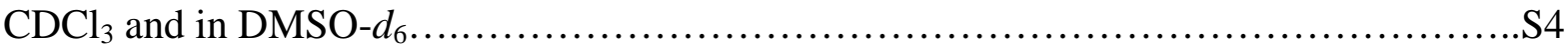

Figure S5: Comparison of the experimental and simulated IR and VCD spectra of

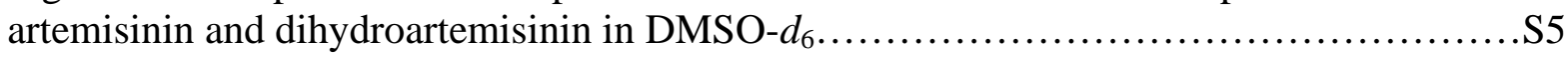

Figure S6: Comparison of the experimental IR and VCD spectra of matrine,

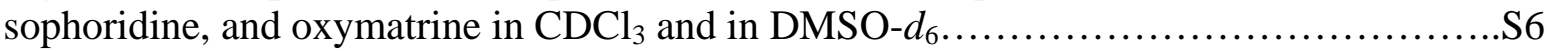

Figure S7: Simulated IR and VCD spectra of the two conformers of the matrine- $\mathrm{CDCl}_{3}$ complex in $\mathrm{CDCl}_{3}$ using the $\mathrm{PCM}$ model.

Figure S8: Simulated IR and VCD spectra of the five conformers of the oxymatrine- $\mathrm{CDCl}_{3}$ hydrogen-bonded complex in $\mathrm{CDCl}_{3}$ using the PCM model............................... 7

Figure S9: Simulated IR and VCD spectra of the seven conformers of sophoridine- $\mathrm{CDCl}_{3}$

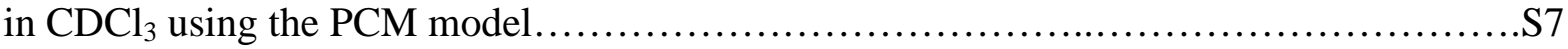

Figure S10: SimIR, SimVCD and SimDF analyses...................................... 
Table S1. The relative energies and Gibbs free energies, and Boltzmann factors of the relevant stable conformers of matrine, oxymatrine, and sophoridine at room temperature at B3LYP/cc-PVTZ level in $\mathrm{CDCl}_{3}$ and DMSO using the PCM model.

\begin{tabular}{|c|c|c|c|c|c|c|c|c|}
\hline & \multicolumn{4}{|c|}{${\text { In } \mathrm{CDCl}_{3}}$} & \multicolumn{4}{c|}{ In DMSO-d } \\
\hline Conformer & $\Delta \mathrm{E}$ & $\mathrm{B}_{\mathrm{f}} \%$ & $\Delta \mathrm{G}$ & $\mathrm{B}_{\mathrm{f}} \%$ & $\Delta \mathrm{E}$ & $\mathrm{B}_{\mathrm{f}} \%$ & $\Delta \mathrm{G}$ & $\mathrm{B}_{\mathrm{f}} \%$ \\
\hline trans-matrine & 0 & 85.0 & 0 & 89.4 & 0 & 84.6 & 0 & 88.9 \\
\hline cis-matrine & 4.30 & 15.0 & 5.30 & 10.6 & 4.23 & 15.4 & 5.16 & 11.1 \\
\hline trans-oxymatrine & 0 & 85.3 & 0 & 88.9 & 0 & 87.3 & 0 & 91.8 \\
\hline cis-oxymatrine & 4.37 & 14.7 & 5.16 & 11.1 & 4.77 & 12.7 & 5.99 & 8.2 \\
\hline sophoridine_I & 0 & 61.9 & 0 & 73.6 & 0 & 60.4 & 0 & 72.3 \\
\hline sophoridine_II & 2.49 & 22.7 & 3.57 & 17.4 & 2.31 & 23.7 & 3.26 & 19.4 \\
\hline sophoridine_III & 4.67 & 9.4 & 7.29 & 3.9 & 4.60 & 9.4 & 7.34 & 3.7 \\
\hline sophoridine_IV & 5.78 & 6.0 & 6.63 & 5.1 & 5.55 & 6.4 & 6.84 & 4.6 \\
\hline
\end{tabular}

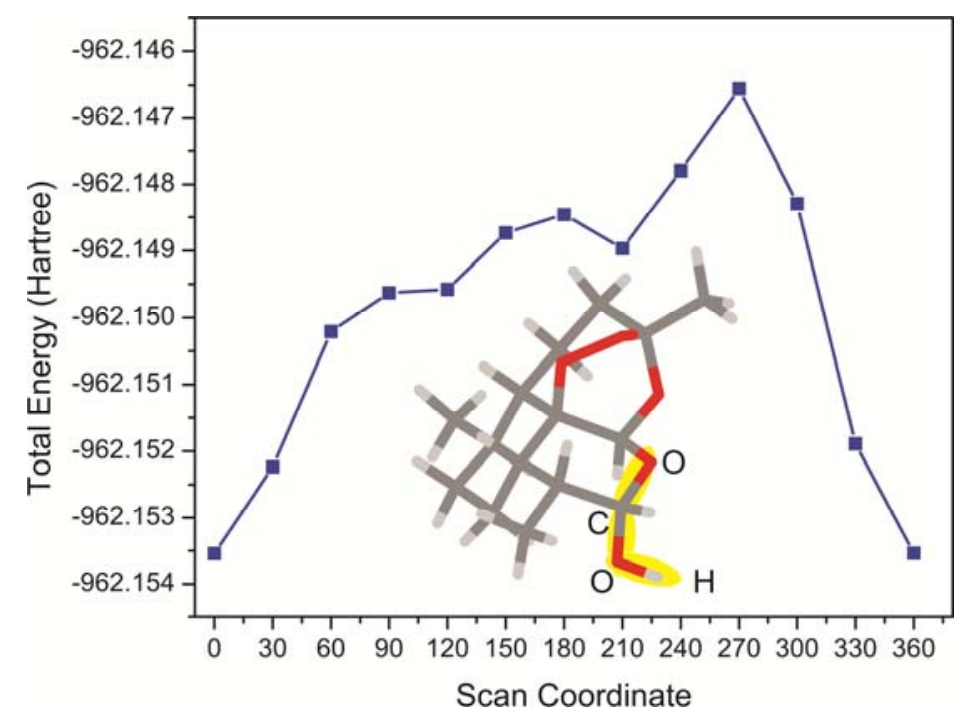

Figure S1. The potential energy profile of dihydroartemisinin along the HOCO dihedral angle highlighted in the figure at the B3LYP/6-31+G(d,p) level. 

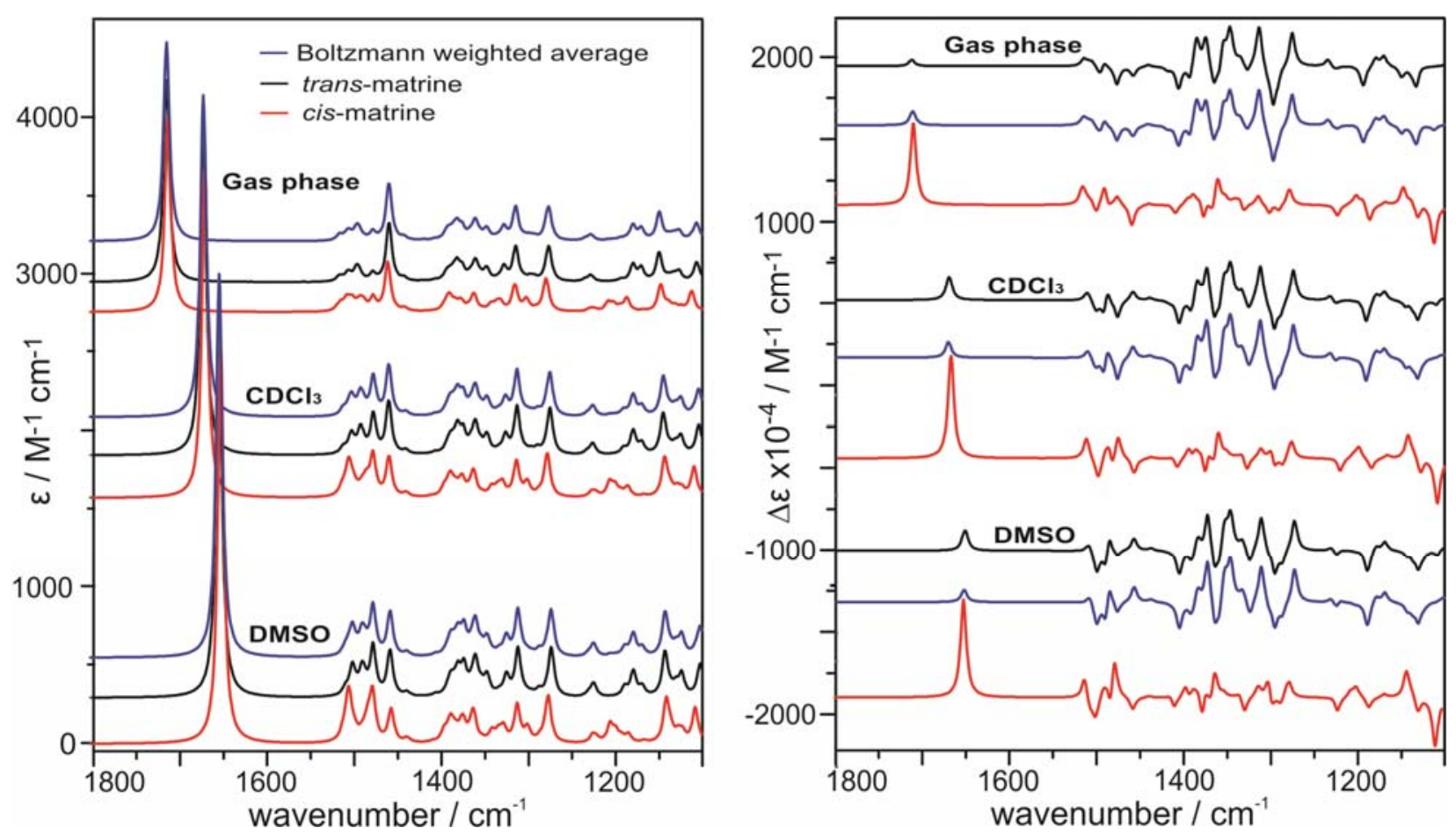

Figure S2. Simulated IR and VCD spectra of the two relevant conformers of matrine at room temperature at the B3LYP/cc-pVTZ level. The PCM model was used for $\mathrm{CDCl}_{3}$ and DMSO- $d_{6}$ solvent.
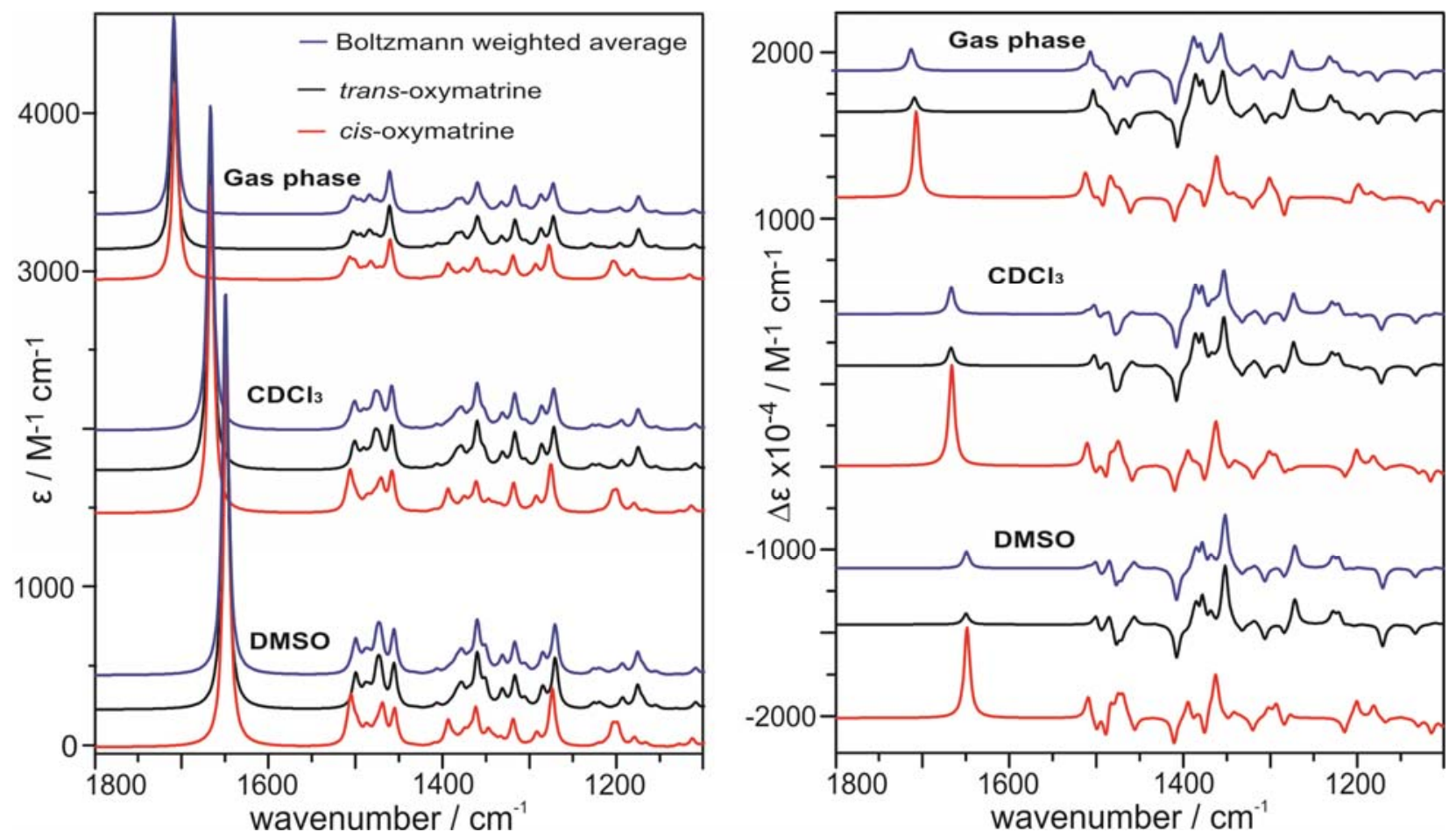

Figure S3. Simulated IR and VCD spectra of the two relevant conformers of oxymatrine at room temperature at the B3LYP/cc-pVTZ level. The PCM model was used for $\mathrm{CDCl}_{3}$ and DMSO- $d_{6}$ solvent. 

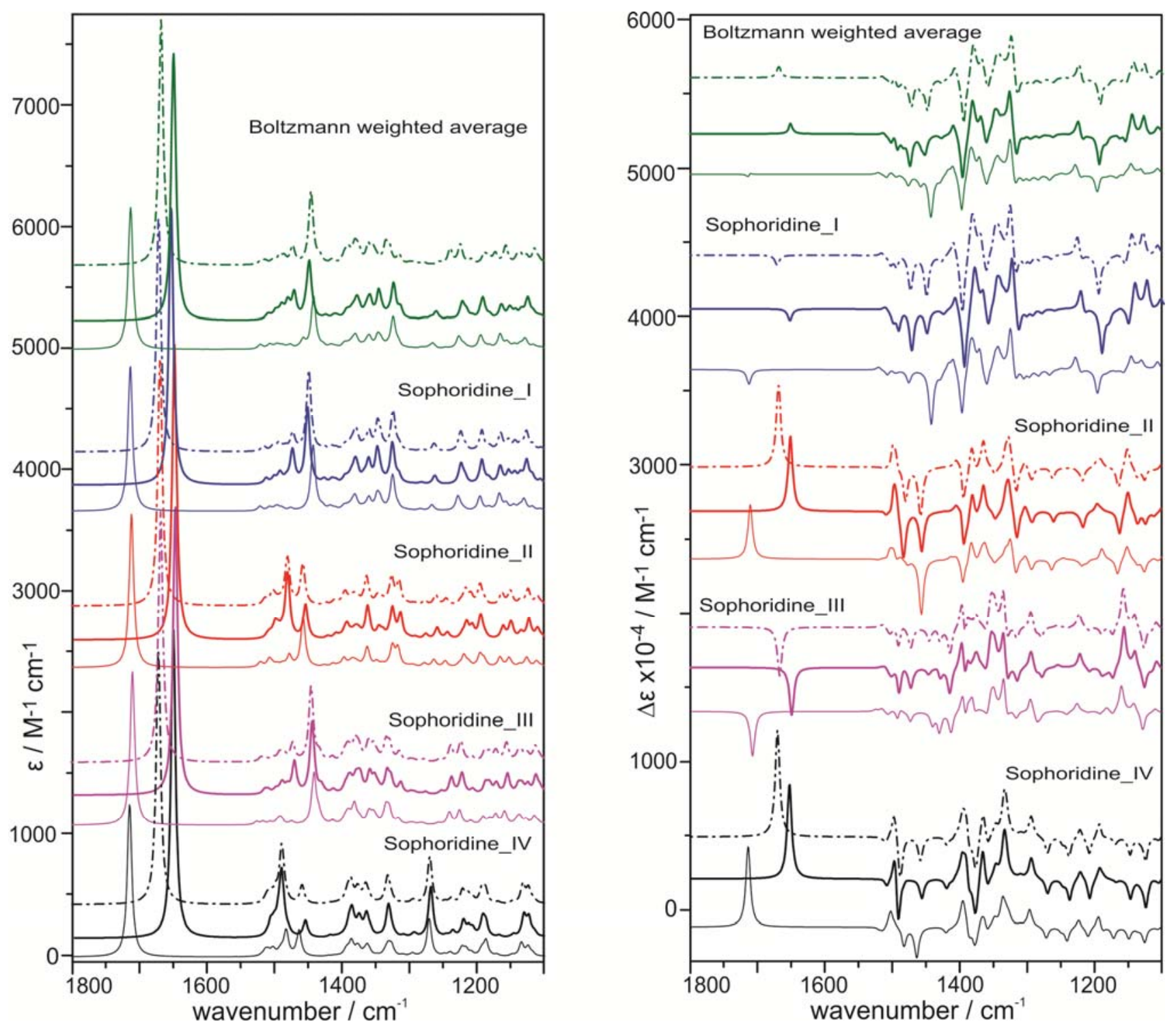

Figure S4. Simulated IR and VCD spectra of the four relevant conformers of sphoridine at room temperature in the gas phase (thin line), in DMSO- $d_{6}$ (thick line), and in $\mathrm{CDCl}_{3}$ (dashed-dotted line) using the PCM model at the B3LYP/cc-pVTZ level. 

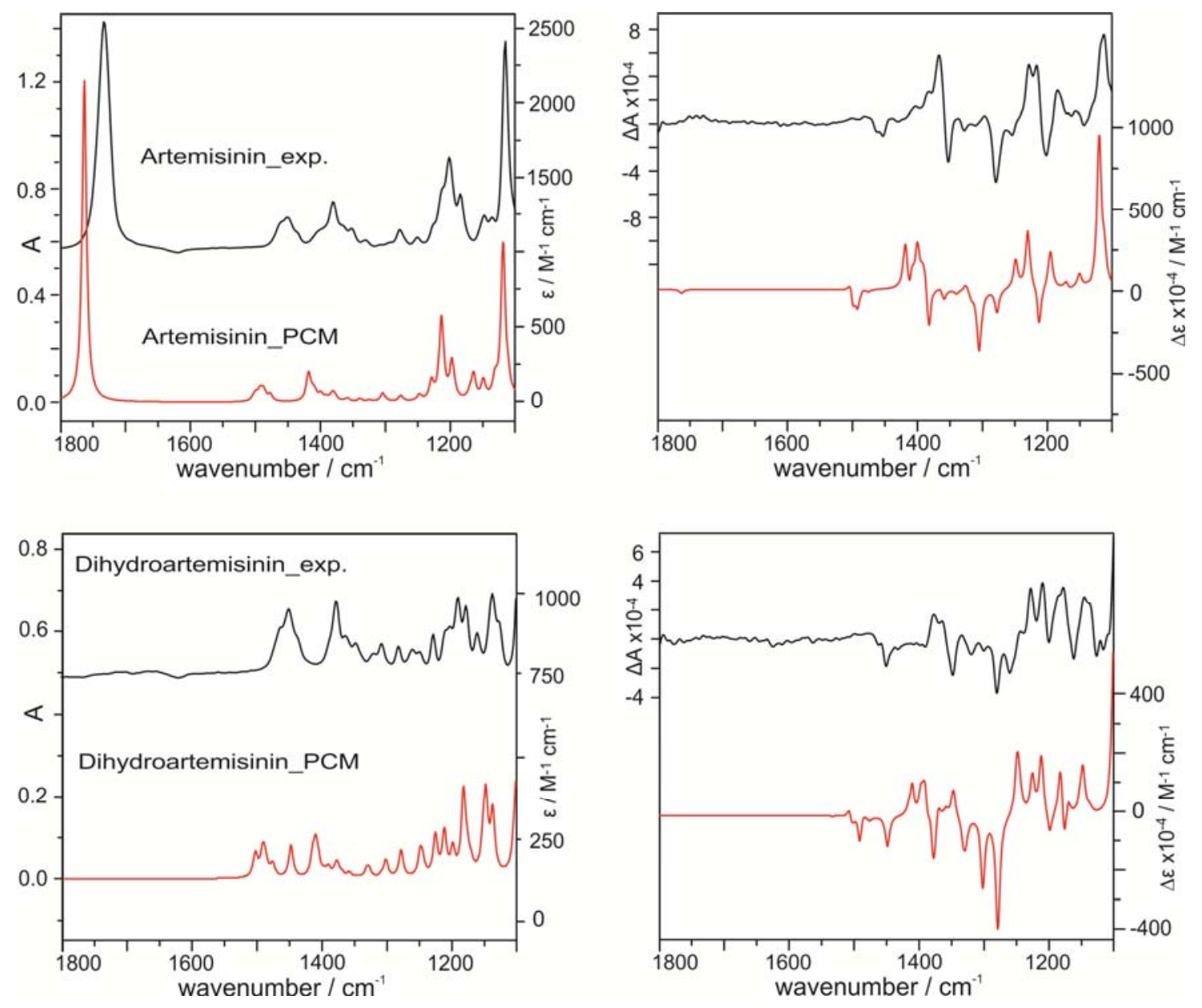

Figure S5. Comparison of the experimental and simulated IR and VCD spectra of artemisinin and dihydroartemisinin in DMSO- $d_{6}$ at the B3LYP/cc-pVTZ level. 

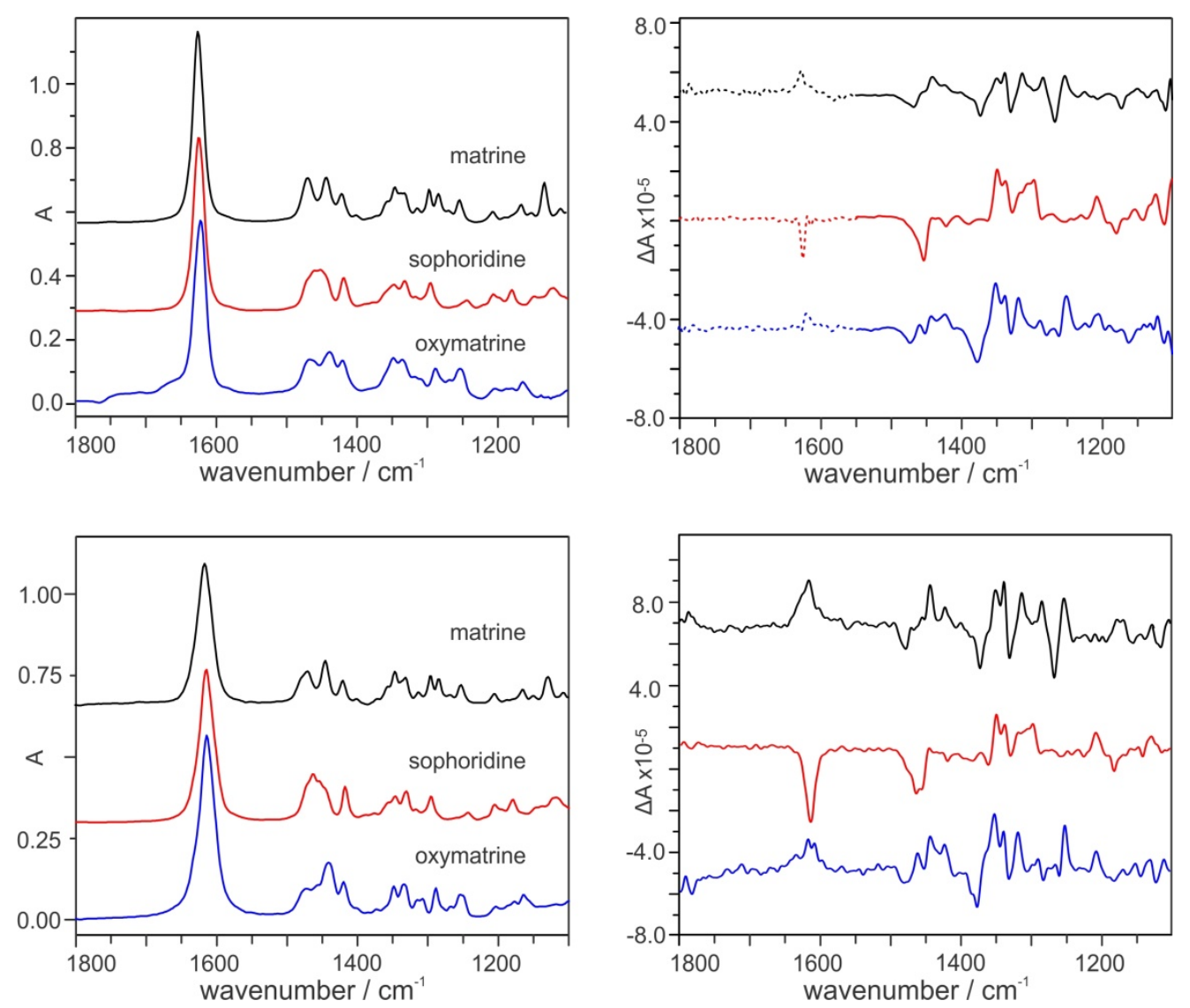

Figure S6. Comparison of the experimental IR and VCD spectra of matrine, sophoridine, and oxymatrine in DMSO- $d_{6}$ (top) and in $\mathrm{CDCl}_{3}$ (bottom).
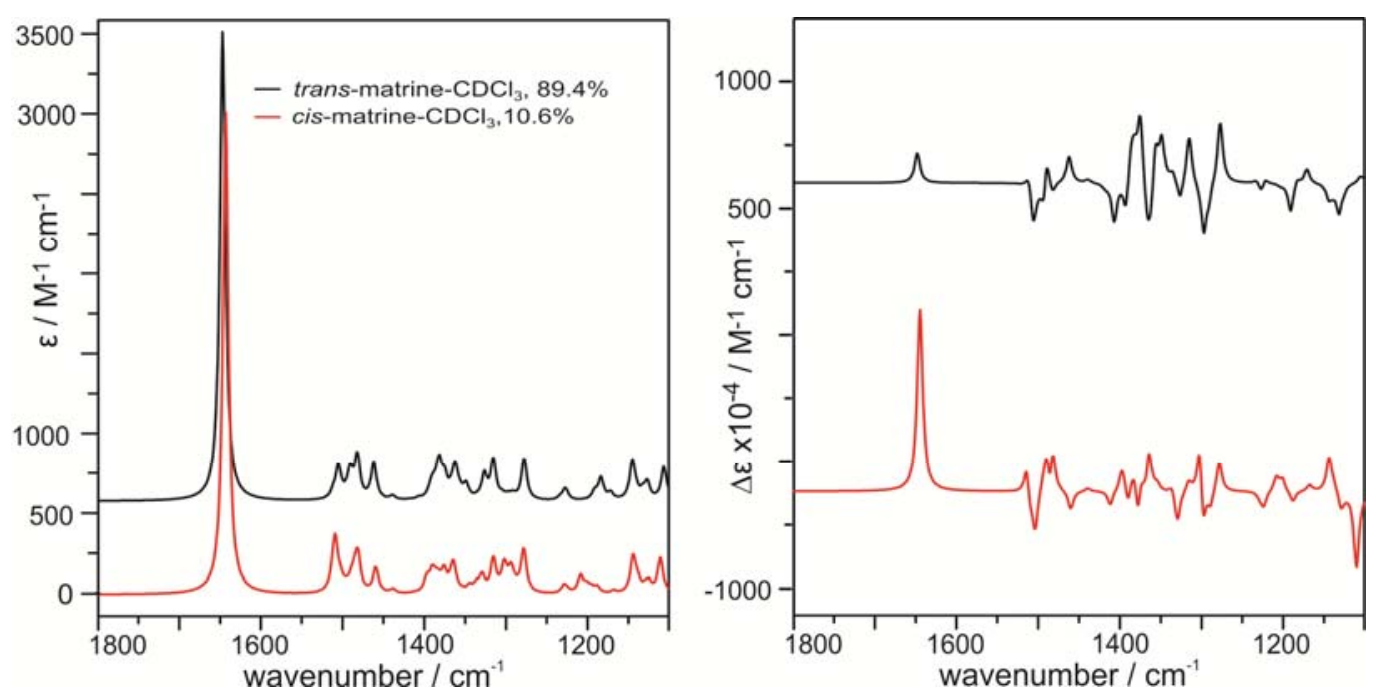

Figure S7. Simulated IR and VCD spectra of the two conformers of the matrine- $\mathrm{CDCl}_{3}$ complex in $\mathrm{CDCl}_{3}$ using the PCM model at the B3LYP/cc-pVTZ level. 

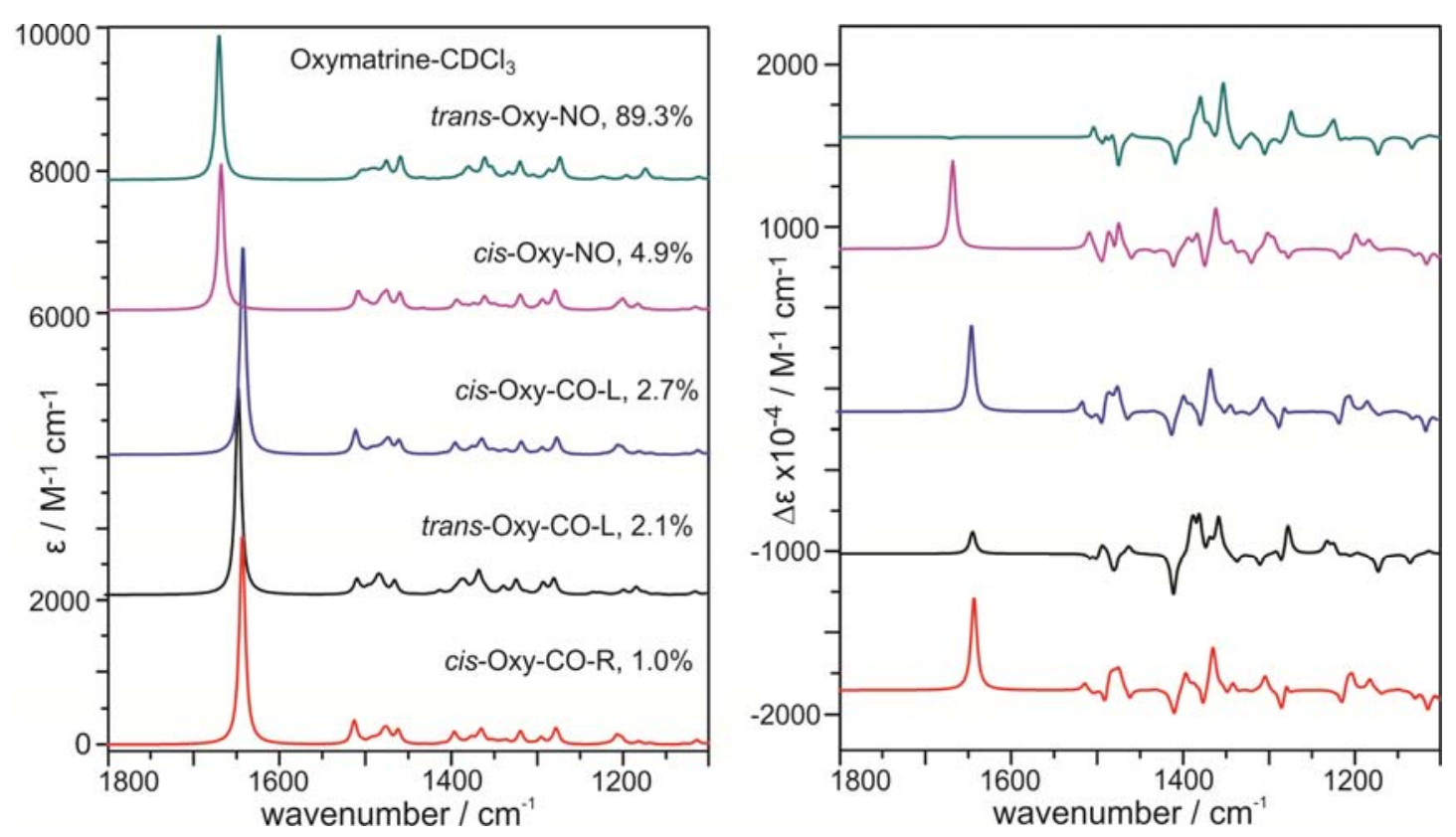

Figure S8. Simulated IR and VCD spectra of the five conformers of the oxymatrine-CDCl $\mathrm{C}_{3}$ hydrogen-bonded complex in $\mathrm{CDCl}_{3}$ using the PCM model at the B3LYP/cc-pVTZ level. I and II refer to trans- and cis-oxymatrine, respectively, while $\mathrm{L}$ or $\mathrm{R}$ indicate $\mathrm{CDCl}_{3}$ approaching the carbonyl O lone pairs from left or right with respect to the matrine frame depicted in Figure 1. "NO" and "CO" denote the hydrogen-bonding sites at the oxide and at the carbonyl position, respectively.
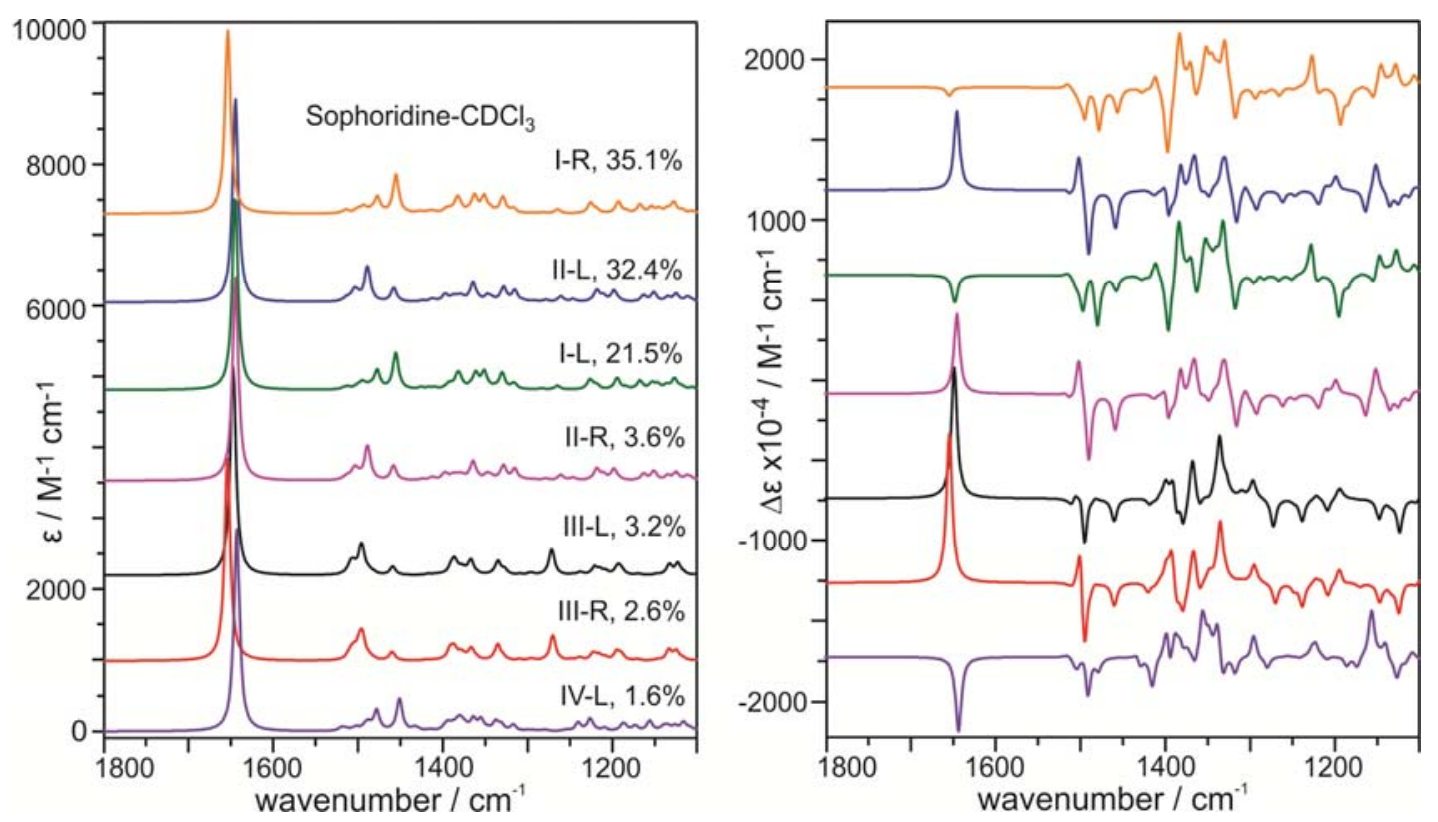

Figure S9. Simulated IR and VCD spectra of the seven conformers of sophoridine-CDCl $\mathrm{CDCl}_{3}$ using the PCM model at the B3LYP/cc-pVTZ level. I, II, III, and IV refer to the conformers of sophoridine identified in Figure $\mathrm{S} 4$, while $\mathrm{L}$ or $\mathrm{R}$ indicate $\mathrm{CDCl}_{3}$ approaching the carbonyl $\mathrm{O}$ lone pairs from left or right with respect to the matrine frame depicted in Figure 1. 
a)
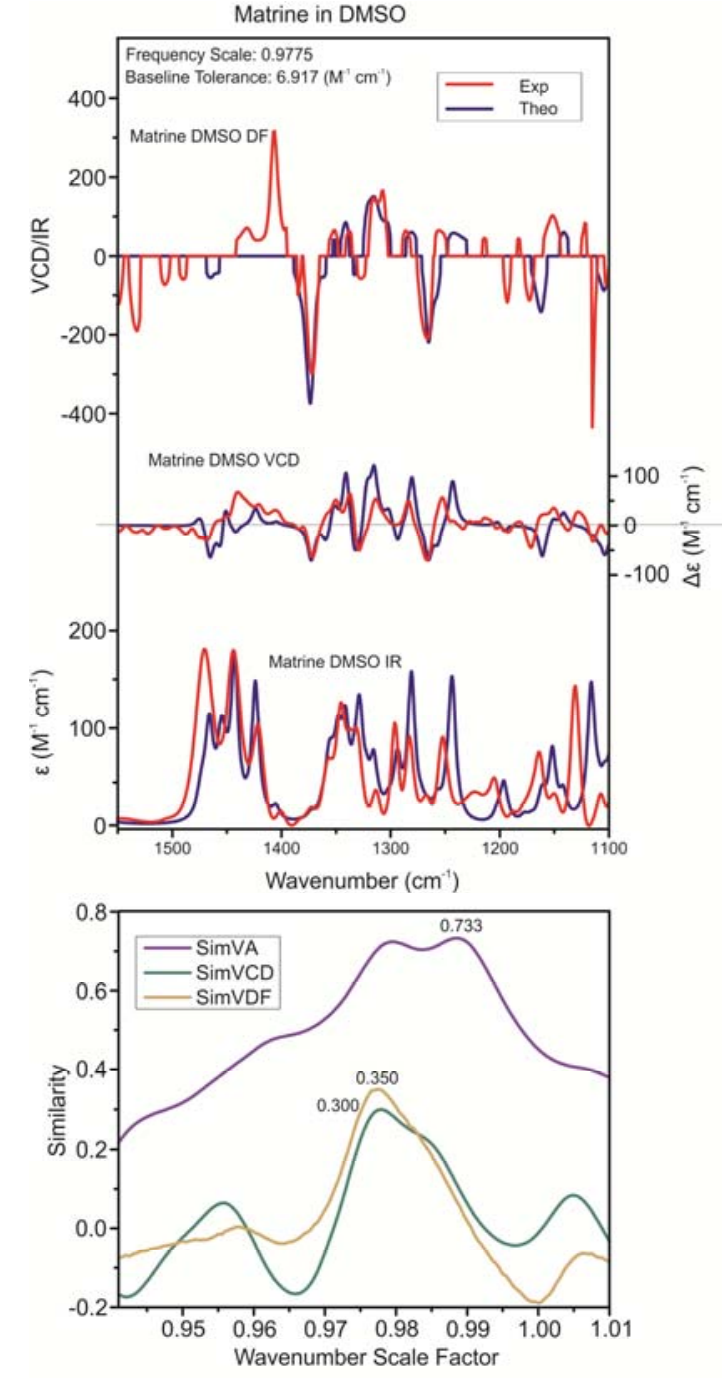
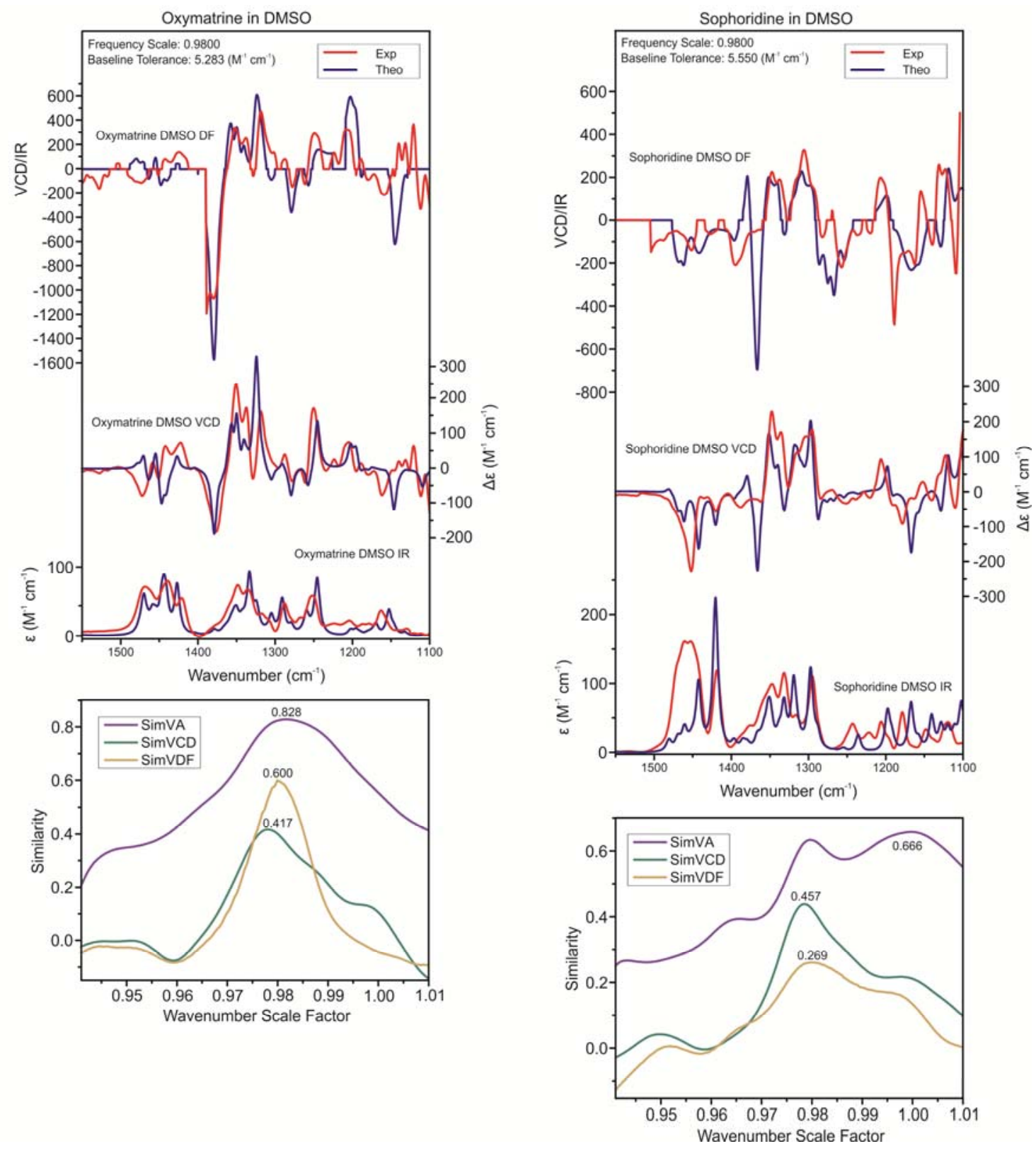

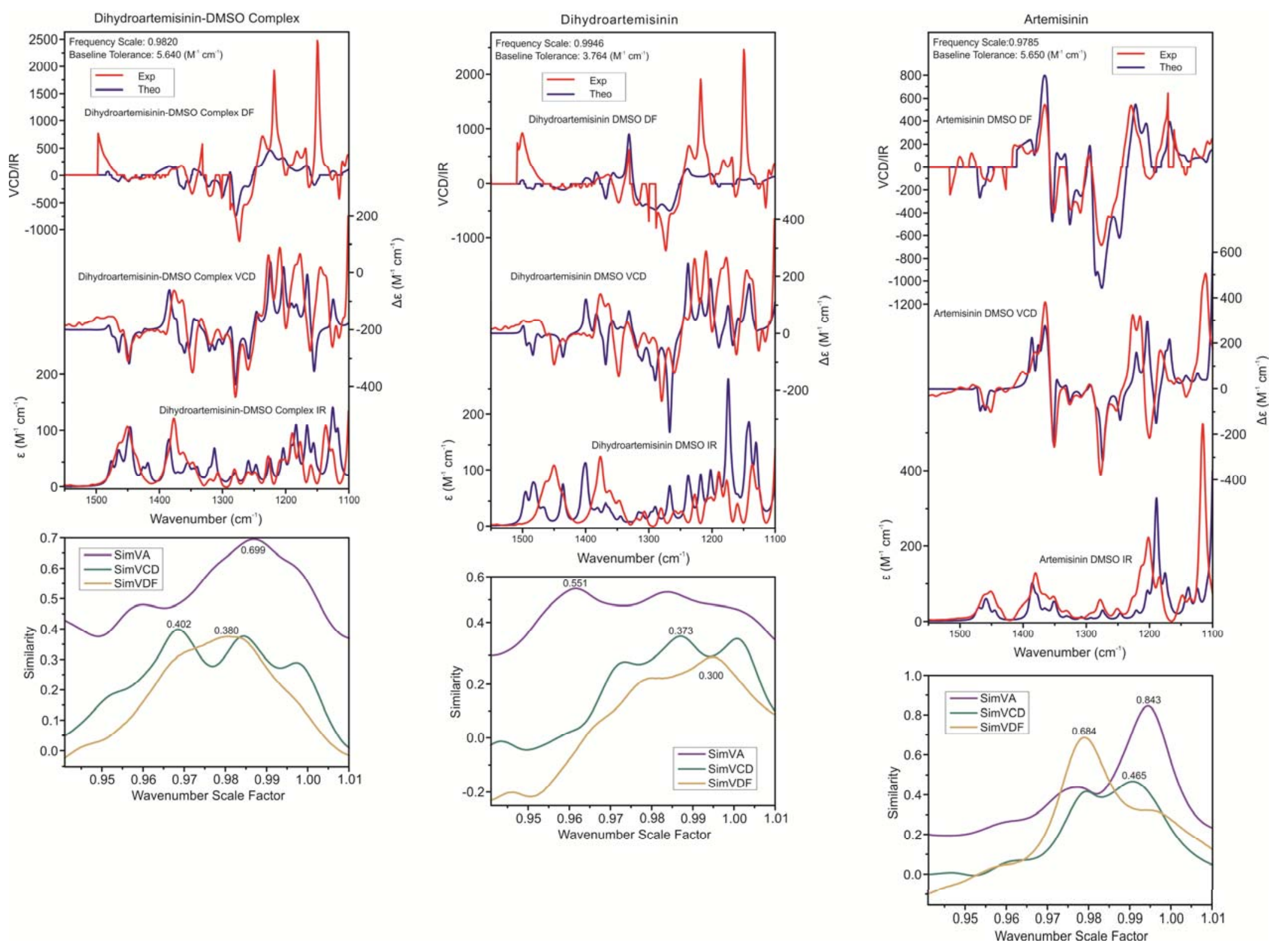
c)
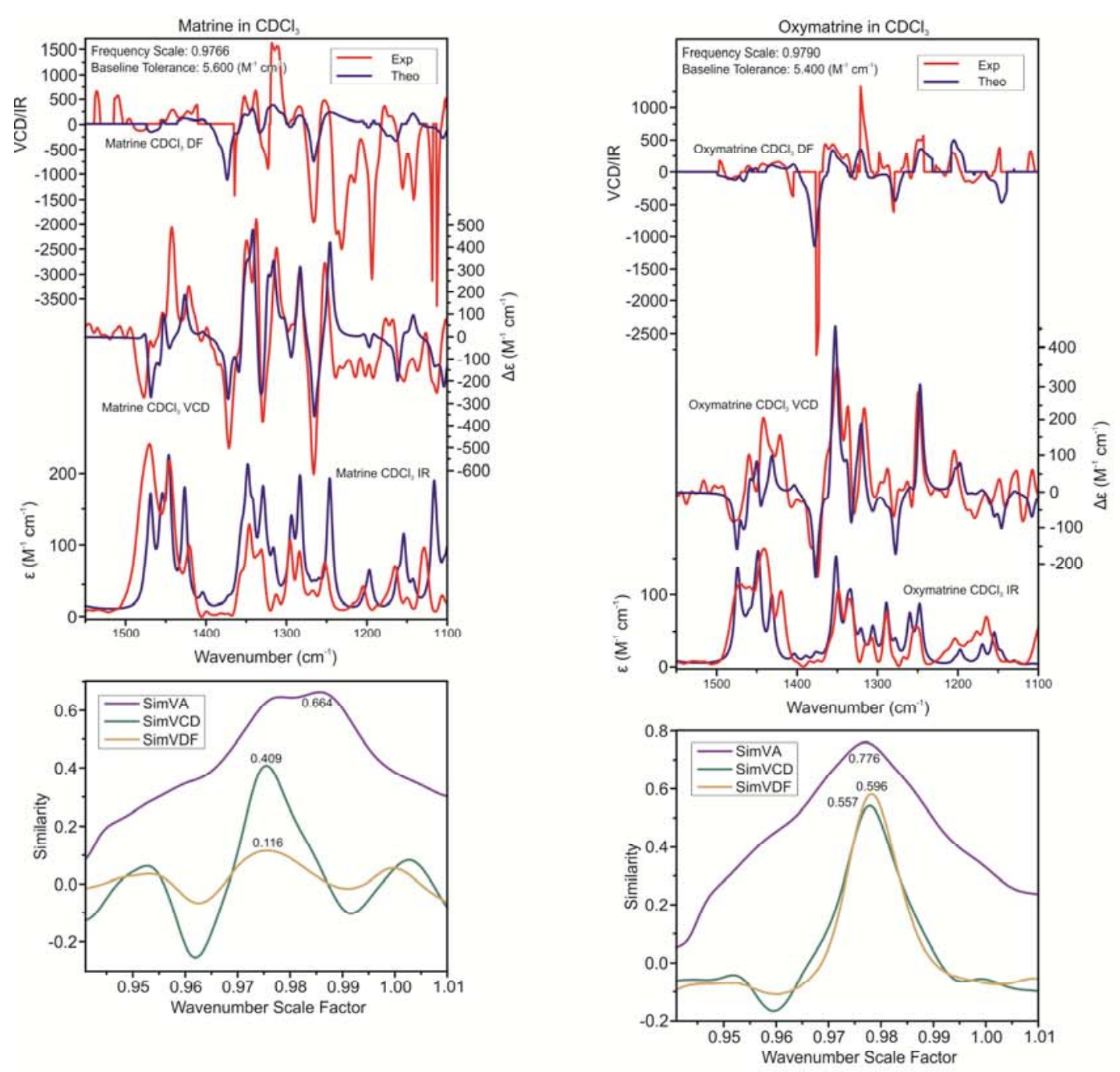
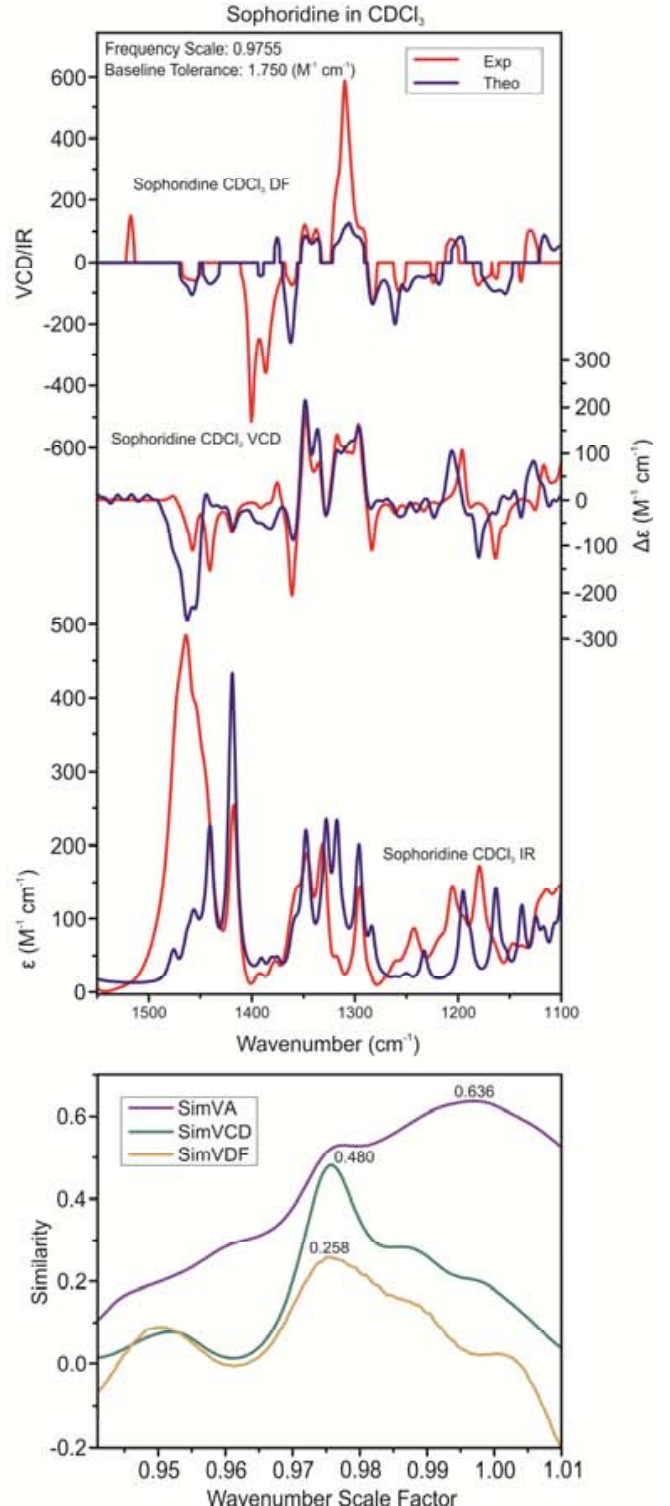

Figure S10. SimIR, SimVCD and SimDF analyses of a) the matrine type compounds in DMSO- $d_{6}$, b) the artemisinin type compounds in DMSO- $d_{6}$, and c) the matrine type compounds in $\mathrm{CDCl}_{3}$. 